\title{
The influence of joints on friction induced vibration in brake squeal
}

\author{
Sebastian Kruse ${ }^{1 \mathrm{a}, \mathrm{b}}$, Merten Tiedemann ${ }^{\mathrm{b}}$, Bernhard Zeumer $^{\mathrm{c}}$, Pascal Reuss ${ }^{\mathrm{c}}$, Hartmut Hetzler $^{\mathrm{d}}$, Norbert Hoffmann ${ }^{\mathrm{b}, \mathrm{e}}$ \\ ${ }^{a}$ Audi AG, Development Foundation Brake, 85045 Ingolstadt, Germany \\ ${ }^{b}$ Hamburg University of Technology, Dynamics Group, 21073 Hamburg, Germany \\ ${ }^{c}$ University of Stuttgart, Institute of Applied and Experimental Mechanics, 70569 Stuttgart, Germany \\ ${ }^{d}$ Karlsruhe Institute of Technology, Institute of Technical Mechanics, 76049 Karlsruhe, Germany \\ ${ }^{e}$ Imperial College London, Department of Mechanical Engineering, London SW7 2AZ, United Kingdom
}

\begin{abstract}
The effect of joints on flutter type friction induced oscillation in friction brakes is investigated. The work is based on a small characteristic system with elementary joint models. Linear stability analysis is conducted and a harmonic balance method is applied to determine limit cycle amplitudes and bifurcation diagrams. It turns out that in finite amplitude oscillation the resulting dynamic state of the joints is dominated largely by the deflection shape of the underlying linear instability. Both local and global bifurcations do exist and large limit cycle amplitudes may result for quite unexpected parameters, depending on the kind of joints involved and related to their dynamical state.
\end{abstract}

Keywords: friction induced vibration, joints, limit cycle approximation, harmonic balance, brake squeal, bifurcations

\section{Introduction}

Brake squeal is a problem that has been challenging engineers in industry for decades. It is commonly agreed that it occurs due to a friction induced oscillation. Numerous works have analyzed the phenomenon of brake squeal, ranging from basic studies on mechanisms up to the development of suitable measurement techniques $[1,2,3]$. Nowadays, there is a strong research focus on numerical simulation $[4,5,6,7]$. Some years ago stability studies in the form of eigenvalue analysis of the linearized system became state of the art. This kind of linear stability analysis is now used broadly in industry to analyze stability borders and to suggest measures against squeal. However, linear stability analysis typically results in a large number of instabilities, while in testing there are way fewer squeal events. There can be several reasons for this problem such as modelling errors or an insufficient implementation of damping effects. Another reasons might be that the limit cycles at those frequencies are of small amplitude so that they are not noticed or recorded in testing. It is obvious that progress can only be made by taking into account bifurcation structures and limit cycle amplitudes [8]. Availability of tools for an analysis of bifurcation structures on industrial scale are still limited though. One of the reasons for this is the numerical effort that would be involved in time-integration approaches. Another reason is the still prevailing lack of knowledge about the nonlinearities actually involved in the systems.

There are definitely several possible sources of nonlinearity in brake systems which could determine finite amplitude vibration levels: nonlinear material characteristics of all components such as the lining material [9], the nonlinearity of the friction interface or the nonlinearity in the joints and contact interfaces of the system [10, 11]. Interestingly, the latter has barely been looked at yet for the phenomenon of brake squeal even though the highly nonlinear behaviour of joints and contact interfaces is well known [12, 13, 14, 15, 16, 17, 18]. As these studies suggest, mechanical joints can generate a wide range of effects. Only recently some first studies based on a single- and a two-degree-of-freedom (DOF) model including self-excitation and dry friction type joints have been conducted [19, 20, 21]. In these studies questions with respect to the existence and characteristics of sets of solutions have been investigated from a discontinuous systems perspective and the question of attractivity of such sets of equilibria has been analysed. Another source of nonlinear system behaviour is thought to originate from stick-slip phenomena in the pad-disc friction interface $[22,23,24,25,26,27]$. Considering the comparably large rotational speeds of the brake disk during typical operational

\footnotetext{
${ }^{1}$ Corresponding author: email: sebastian.kruse@ audi.de
} 
squeal conditions, these effects are however thought to be unlikely to play a significant role [28]. For numerical modeling and simulation a number of techniques have been proposed to approximate limit cycles for brake oscillations in large scale systems. A recent overview is for example given in [29]. From the point of view of numerical efficiency, Harmonic Balance (HB) approaches [30] are now mostly adapted and can be used on large-scale finite element based models if the number of nonlinear degrees of freedom in the model does not get too excessive [31].

At the moment one of the most pressing questions does still seem to be the lack of deeper insight about the dynamics of friction self-excited vibrations with mechanical joints involved in the system. Thus, the aim of the present study is to better understand how joints may affect bifurcations in such systems, and to find out how joints influence limit cycle amplitudes. We analyze the influence of joints on friction induced vibrations with the help of so called whole-joint models, i.e. the whole-joint is represented by a single dynamical equation [15], and a minimal model prone to friction induced oscillation. We assume that the time-scales of processes in the frictional interface can be separated from the time-scales of the structural dynamics, as it is usually done in studies focusing on flutter type instabilities $[32,33,34,35]$. Following up earlier work [19, 20, 21], we focus on the role that joint characteristics have on limit cycle amplitudes and bifurcation structures. As a result, it will be shown subsequently how joints can be activated or deactivated, depending on the dynamical state, and how this may lead to interesting bifurcations structures, i.e. bifurcations from infinity, detached branches, i.e. isola, and unexpected limit cycle amplitudes in general.

The paper is structured as follows. First, the characteristic model under study is presented, the joint models to be investigated are introduced, and the numerical approaches chosen are described. Then the findings on bifurcations in the model are reported and discussed with respect to the interplay of friction-induced self-excitation and joint dynamics. Finally, we draw conclusions and provide an outlook to future research.

\section{Modeling and numerical approach}

\subsection{Model system with joint}

In order to discuss the influence of joints on friction induced flutter a minimal model is analyzed. The system is an extension of the one used in [36]: A simple substructure is added via a joint. The model thus comprises a module showing friction induced flutter, a module with further structural components, and a joint in between. A graphical interpretation is given in Fig. 1a and Fig. 1b, each including a different joint model.

a)

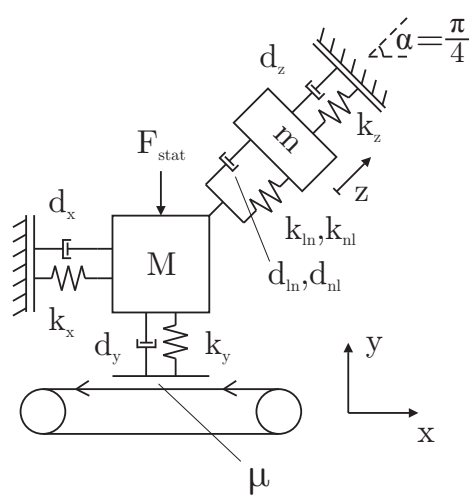

b)

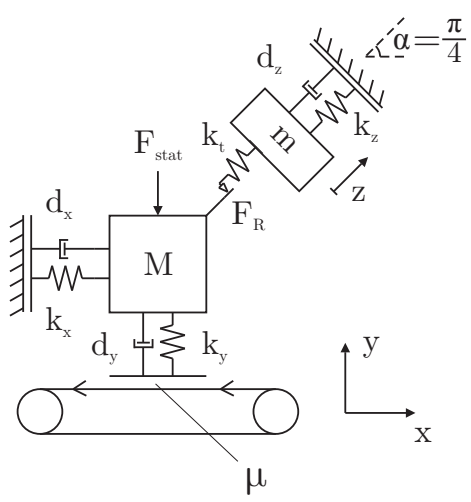

Figure 1: Model systems with polynomial stiffness type joint in a) and elasto-slip type joint in b).

So-called whole-joint models are a widespread approach to capture joint dynamics in numerical simulation of large structures with small contact interfaces [15]. According to this, the dynamic behaviour of the whole joint is described by an evolution equation. Following this approach the first joint model from Fig. 1 a) is chosen to capture elementary properties of joints with smooth but nonlinear stiffness behaviour. For that purpose a simple nonlinear polynomial stiffness characteristic is assumed. The constitutive equation of this joint model is given by

$$
F_{n l}=k_{l n} \cdot u+k_{n l} \cdot u^{3}+d_{l n} \cdot \dot{u},
$$


where $F_{n l}, k_{l n}, d_{l n}$ and $k_{n l}$ are the full nonlinear joint force, the linear joint stiffness coefficient, the linear joint viscous damping coefficient, and the cubic joint stiffness coefficient, respectively. In the remainder of this paper this polynomial nonlinearity, representing joint behaviour, will be referred to the polynomial type joint.

The second kind of joint from Fig. $1 \mathrm{~b}$ is a simple elasto-slip model $[13,16]$. This joint model consists of a linear spring and a dry friction element in series. Thus, it basically behaves like a linear spring when small tangential forces are applied to a stick state, whereas it acts like a Coulomb sliding friction damper when the forces on the joint exceed a certain limit. Though oversimplifying, it may be taken as a representative for joints including dry friction.

Based on [13] the dynamics of this joint can be described by

$$
F_{n l}=\left\{\begin{array}{ll}
k_{t} \cdot u & \text { if }|u|<\frac{F_{R}}{k_{t}} \\
F_{R} \cdot \operatorname{sgn} \dot{u} & \text { if }|u| \geq \frac{F_{R}}{k_{t}}
\end{array},\right.
$$

where $u, \dot{u}, F_{R}, k_{t}$ are the deflection in the joint, its derivative, the threshold force and the tangential stiffness, respectively. Following [16] this model will be referred to as elasto-slip joint. With these two elementary joint models a wide range of joint types is covered for the following study of their effects on flutter type instabilities in systems with frictional contacts.

Based on the description of the whole model and of the joints, altogether the resulting evolution equations for the system can be written in the notation usually used in nonlinear dynamics as

$$
\mathbf{M} \cdot \ddot{\mathbf{x}}(t)+(\mathbf{D}+\mathbf{G}) \cdot \dot{\mathbf{x}}(t)+(\mathbf{K}+\mathbf{N}) \cdot \mathbf{x}(t)+\mathbf{f}_{\mathbf{n l}}(\mathbf{x}, \dot{\mathbf{x}}, t)=\mathbf{f}_{\mathbf{e x t}}(t),
$$

where the vector of deflections $\mathbf{x}(t) \in \mathbb{R}^{N}$, the vector of nonlinear restoring forces $\mathbf{f}_{\mathbf{n}} \in \mathbb{R}^{N}$ and the vector of external forces $\mathbf{f}_{\text {ext }} \in \mathbb{R}^{N}$ are defined as

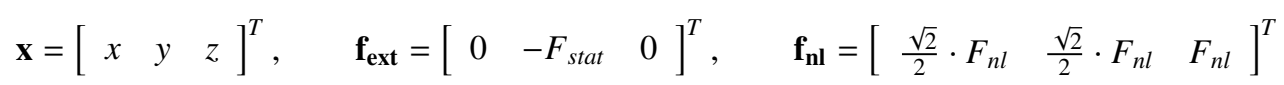

and $N, F_{\text {stat }}$ and $F_{n l}$ are the number of states, the static external force and the nonlinear force in the joint respectively. The matrices $\mathbf{M}, \mathbf{D}, \mathbf{G}, \mathbf{K}$ and $\mathbf{N} \in \mathbb{R}^{N \times N}$ resulting from the described model, and also the actually applied numerical values, can be found in Appendix A. For simplicity, we assume non pre-stressed joints for the static or steady sliding state equilibrium solutions of the model. In finite element analysis of systems subject to friction-induced vibrations another notation of the equation of motion is commonly used, where the displacement-dependent terms are collected in an elastic stiffness and a contact stiffness matrix derived by linearization of the contact forces in the pad-disc interface. In this context, the elastic stiffness matrix is employed in the necessary model order reduction step. However, for the studied system this notation is not more advantageous than the one used in equation (3).

\subsection{Linear stability analysis}

The stability of the equilibrium solution is investigated by an eigenvalue analysis. The linearized equations of motion, based on equation (3), are treated as an eigenproblem with the eigenvalues $\lambda_{i}$ and corresponding right eigenvectors that represent modal shapes. The linearization of the nonlinear restoring forces results for both joint models in an additional stiffness term $k_{\text {lin }}$. The equilibrium solution's stability is determined by $\operatorname{sgn}\left(\mathfrak{R}\left(\lambda_{i}\right)\right)$. A positive $\mathfrak{R}\left(\lambda_{i}\right)$ leads to an unstable equilibrium solution and growing vibrational amplitudes, whereas a negative $\mathfrak{R}\left(\lambda_{i}\right)$ leads to a stable equilibrium solution.

The study of linear stability employs a set of parameters given in Appendix B. It is chosen such that for both joint models the linearized equation of motion are identical and such that exactly one eigenvalue $\lambda_{j}$ of the linearized system shows a positive real part, i.e. the system's equilibrium solution turns unstable. Fig. 2a shows the development of the eigenvalues' imaginary parts correlating with the system's resonance frequencies for a variation of the friction level $\mu$. Fig. $2 \mathrm{~b}$ presents the real parts of the eigenvalues which indicate stability or instability. Fig. 2 shows that stability of the equilibrium solution is lost for values of $\mu>0.45=\mu_{H P}$, where $\mu_{H P}$ defines the critical friction level leading to an oscillatory instability. Thus, $\mu_{H P}$ indicates a Hopf Point (HP). 


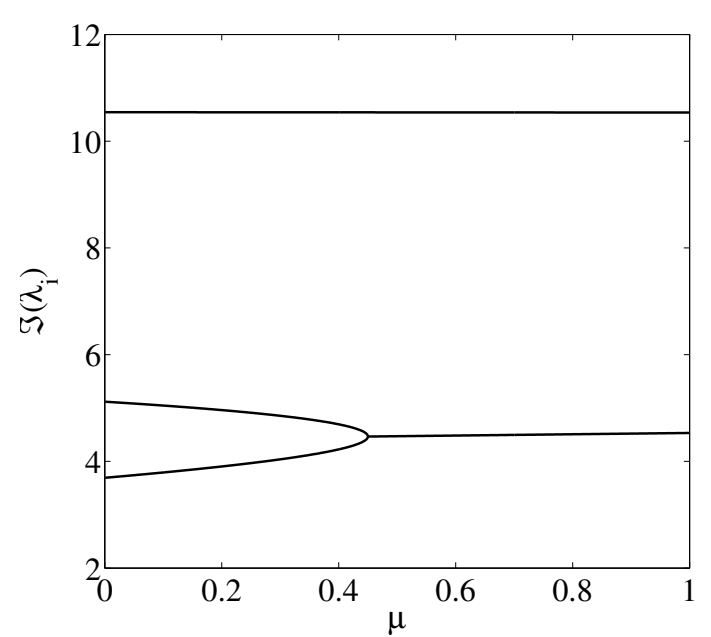

(a)

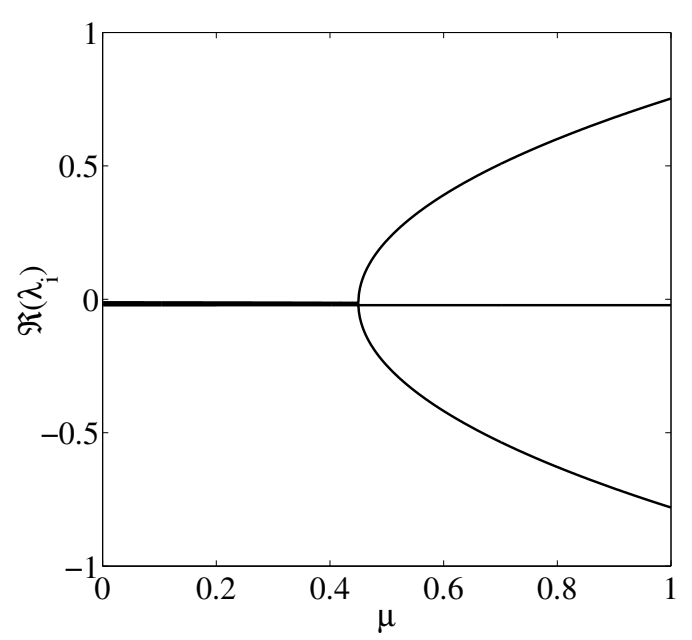

(b)

Figure 2: Development of eigenvalues with bifurcation parameter $\mu$ for parameters given in Appendix B. Imaginary parts (a), and real parts (b).

As already emphasized, HPs mark the linear border of stability. Consequently, the calculation of Hopf curves as functions of different parameters delivers insight into regions of stable and unstable equilibrium solutions as well as their growth or shrinkage depending on the parameters. Fig. 3 shows Hopf curves for different friction levels $\mu$ in the $k_{x}-k_{\text {lin }}$-plane. $k_{x}$ represents a typical system parameter, whereas $k_{\text {lin }}$ is a parameter which indicates the influence of the linearized joint dynamics on the stability behaviour of the equilibrium solution. In this case, regions of unstable equilibrium solutions are encircled by Hopf curves.

The results clearly reveal that the regions of unstable equilibrium solutions grow for higher friction levels. However, even for high friction levels the instability region is finite. Thus, e.g. for suffiently high values of $k_{x}$ finite disturbances should decay. This point will be challenged in this work. In addition, the results illustrate that both, system parameters and joint parameters, influence the stability of the equilibrium solution.

\subsection{Numerical approach for studying the bifurcation behaviour of periodic solutions}

To study the post-flutter bifurcation behaviour of the model system with a joint, the nonlinear equation of motion (3) has to be considered. Among the variety of analytical and numerical methods described in literature to calculate periodic solutions of nonlinear systems few of them seem to be feasible for studying the bifurcation behaviour of large-scale systems. Although in this contribution a minimal model is studied to establish understanding of the basic effects, large-scale system will be studied in the future. Consequently, to make numerical experiments scalable for future work based on large-scale systems a HB approximation technique is applied to determine periodic solutions. For this purpose, the nonlinear restoring force $\mathbf{f}_{\mathbf{n l}}(\mathbf{x}, \dot{\mathbf{x}}, t)$ from equation (3) is expressed as a Fourier series. This approach is wide-spread in nonlinear dynamics and is presented in [30]. Coudreyras et al. applied it to self-excited vibrations of nonlinear systems [31]. The scalability of approaches using the HB method with or without reduction techniques (e.g. component mode synthesis [37]) is discussed and shown in [31, 38, 39, 40]. Besides the scalability of the HB method, these works show the method's ability to handle few, but arbitrary nonlinearities for large FE-models. This aspect further motivates the choice of this method for the present work. Recent experimental studies suggest that there may be only very few dominant or relevant joints in friction brake systems [10]. An extension of the HB method to multi-instabilities in frictional excited systems can be found in [41]. An extension to multi-instabilities is, however, not scope of this work and remains an open point for future analysis.

Applying the above discussed HB technique to the present system, the steady-state solution of the equation of motion (3) can be approximated by 


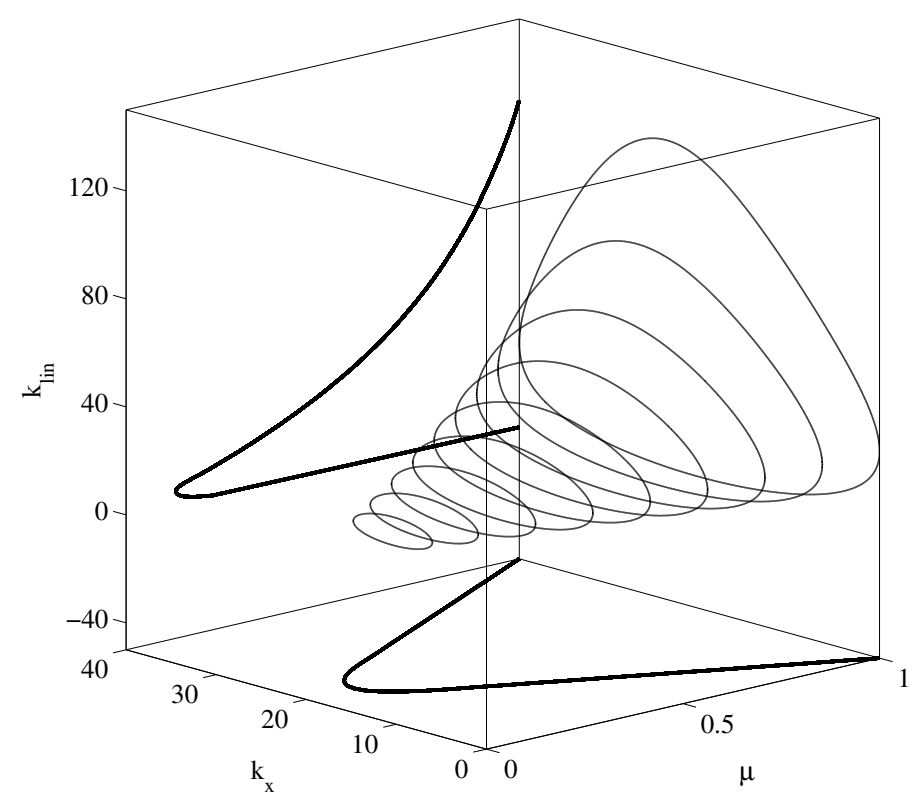

Figure 3: Hopf curves in $k_{x}-k_{\text {lin }}$-plane for different friction levels $\mu$. In addition, the projections on the $k_{x}-\mu$-plane and on the $k_{\text {lin }}-\mu$-plane are displayed.

$$
\mathbf{x}(t) \approx \hat{\mathbf{x}}_{\mathbf{0}}+\sum_{m=1}^{m_{h}}\left(\hat{\mathbf{x}}_{\mathbf{c}, \mathbf{m}} \cdot \cos (m \omega t)+\hat{\mathbf{x}}_{\mathbf{s}, \mathbf{m}} \cdot \sin (m \omega t)\right),
$$

where $m_{h}, \hat{\mathbf{x}}_{\mathbf{0}} \in \mathbb{R}^{N}, \hat{\mathbf{x}}_{\mathbf{c}, \mathbf{m}} \in \mathbb{R}^{N}, \hat{\mathbf{x}}_{\mathbf{s}, \mathbf{m}} \in \mathbb{R}^{N}$ and $\omega$ are the number of harmonics considered, the vectors containing the Fourier coefficients and the unknown limit cycle frequency, respectively. The implementation is described in detail in Appendix C.

To reveal suitability of the employed HB approach, different approximation methods and choices for $m_{h}$ are compared. For this purpose, the periodic solutions bifurcating from the HP detected in the linear stability analysis with the friction level $\mu$ as bifurcation parameter are studied. The solutions are approximated employing orthogonal collocation in combination with well-established path following techniques (CL MatCont [42]) and the HB approach presented in Appendix C. For the latter, the first harmonic $\left(m_{h}=\{1\}\right)$ and the first three odd harmonics $\left(m_{h}=\{1,3,5\}\right)$ are used. To visualize the dependency of solutions on the chosen bifurcation parameters, this work employs the vibration amplitudes $x_{\text {amp }}, y_{\text {amp }}$ and $z_{\text {amp }}$ being the amplitudes of the periodic solution in $x, y, z$-direction, respectively. For the sake of completeness, we want to highlight that from an industrial application point of view considering mechanical energies is also a promising choice to assess the numerical results. This idea will be pursued in future when larger systems are studied. Fig. 4 shows the results of the study. Fig. $4 \mathrm{~b}$ is a detail of Fig. $4 \mathrm{a}$ focusing on the differences in results of the employed methods with a growing distance from the HP. The results reveal that in the neighbourhood of the HP all three approaches are capable of describing the branching behaviour of the periodic solution in a qualitatively and quantitatively satisfying manner. However, as also expected from a theoretical point of view, with increasing distance to the HP the branch approximated with the HB approach using the first harmonic only diverges from the other two. Nevertheless, a qualitative approximation of the branching behaviour seems feasible using one harmonic only. Hence, in the remainder of this work, aiming to generate basic insights regarding the influence of joints on friction induced vibrations, the $\mathrm{HB}$ method as presented in Appendix $\mathrm{C}$ using the first harmonic is employed. This approach should moreover be sufficient for an extension to larger models with weak nonlinear character in future work. One should note, however, that the employed approach might be limited with respect to quantitative accuracy if 
results for parameter sets far away from a HP are of interest.

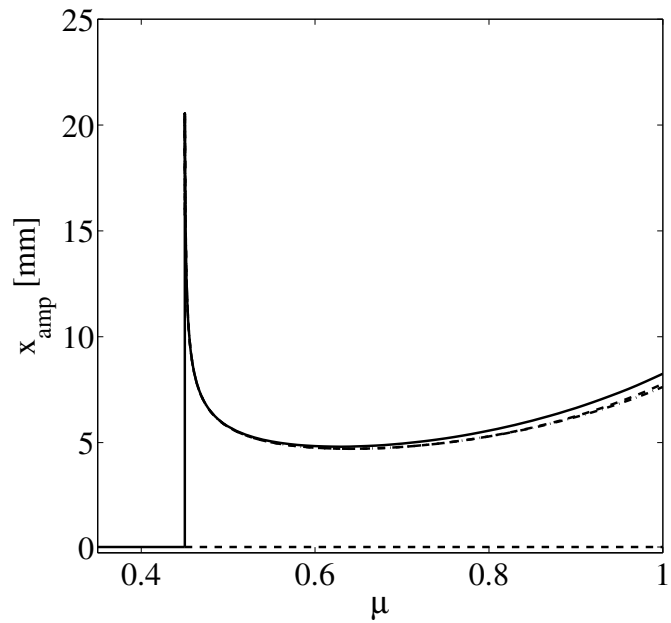

(a)

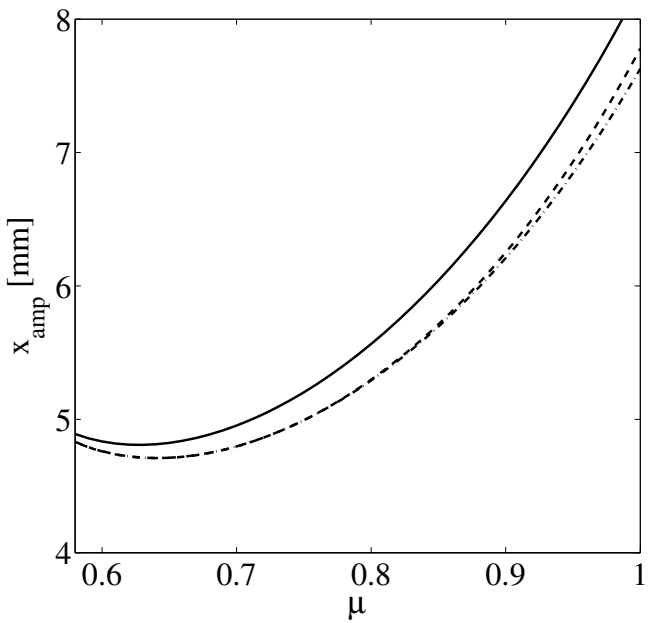

(b)

Figure 4: (a) Comparison of the results of a harmonic balance using one harmonic (solid line), the first three odd harmonics (dashed line) and orthogonal collocation (dashed-dotted line). The stability of the equilibrium solution is indicated by a solid line for stable solutions and by a dashed line for unstable solutions at zero amplitude level. (b) is a detail of (a) focusing on the comparison of the results for higher friction levels.

For a thorough bifurcation analysis not only the periodic solutions have to be calculated but also their stability has to be determined. The HB approach does not directly allow to determine if the obtained periodic solution is stable or unstable. Due to its nature the approach determines both stable and unstable periodic solutions. The stability of periodic solutions can e.g. be determined by a Floquet analysis [30]. In this work, stability is estimated based on the geometrical features of the periodic solution curves and on the direction in which the branch bifurcates from the HP. In fact, in the neighbourhood of a HP stable periodic solutions encircle unstable equilibrium solutions and vice versa. In addition, it is assumed that turning points separate stable solutions from unstable solutions.

\subsection{Open questions}

Revisiting Fig. 4a with a focus on the bifurcation behaviour reveals that the bifurcation diagram for the amplitudes does not look like the typical super- or subcritical continuous bifurcation diagrams that one would expect. The amplitudes do not seem to grow continuously with the distance from the stability border; rather, the largest amplitudes seem to appear right next to the HP. The main purpose of the present study is thus to shed further light on this seemingly unusual behaviour. Detailed studies will clarify what is actually happening and try to give answers to the following questions:

- How well do the borders of linear stability agree with the parameter ranges in which nonlinear solutions, i.e. limit cycles, exist?

- How may different types of joints influence the resulting limit cycle amplitudes?

- Can the bifurcation structure and limit cycle behaviour be understood in terms of some properties already inherent in the linear stability analysis, like e.g. the eigenvectors? 


\section{Post-flutter bifurcation analysis}

\subsection{The dynamics of joints as the dominant factor for limit cycle amplitudes}

First, the model introduced in Fig. 1a including the polynomial type joint is studied. The results from linear stability analysis indicate that for a wide range of parameters two HPs can be found by a variation of $k_{x}$, i.e. points from which branches of periodic solutions bifurcate. Whether the bifurcation is supercritical or subcritical depends obviously on the nature of the nonlinearity which is involved, in this case on the parameters of the polynomial type joint. For this reason, a bifurcation study in the neighbourhood of a HP is conducted with $k_{x}$ as control parameter. Furthermore, the cubic stiffness characteristic is assumed to vary from degressive via linear to progressive. The results are presented in Fig. 5.

a)

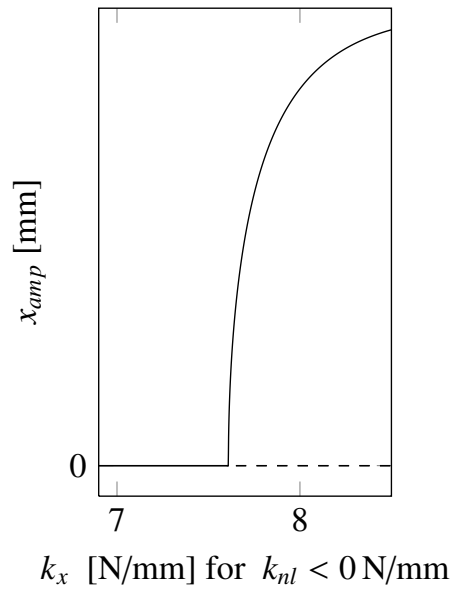

b)

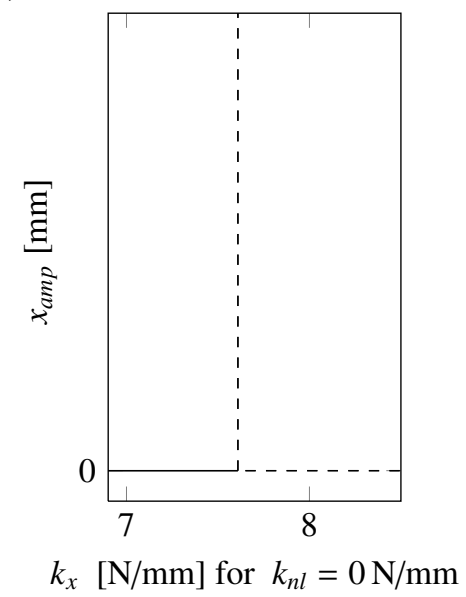

c)

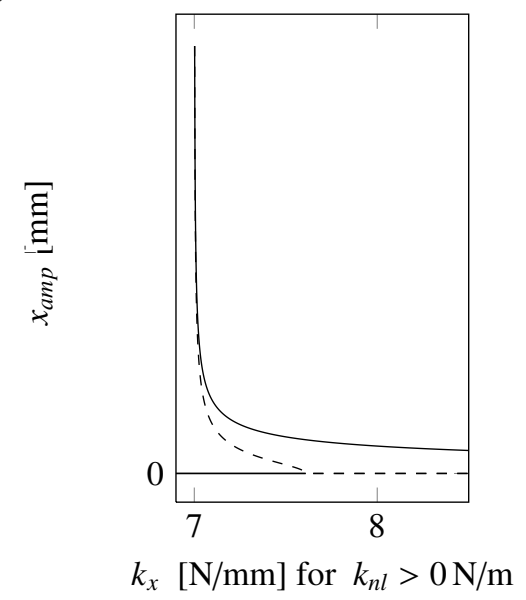

Figure 5: Transition from supercritical to subcritical Hopf-bifurcation triggered by $k_{n l}$. Stable equilibrium and periodic solutions are plotted as solid lines, unstable solutions as dashed lines.

The central plot shows the linear behaviour of the model leading to unlimited amplitudes. A weakening behaviour of the cubic joint with $k_{n l}<0$ produces the generic behaviour of a supercritical bifurcation, see Fig. 5a. A different behaviour is observed for a stiffening joint characteristic. For a cubic joint with $k_{n l}>0$ a subcritical Hopf-bifurcation arises. This causes areas in the parameter range where the equilibrium solution is stable, but a finite disturbance of that state may lead to limit cycle oscillations. Fig. 6 gives results for $k_{n l}>0$ when $k_{x}$ varies over larger ranges to obtain a more complete picture.

The linearized model shows two HPs, at around $k_{x}=7.6 \mathrm{~N} / \mathrm{mm}$ and $k_{x}=32.3 \mathrm{~N} / \mathrm{mm}$. Between these HPs the system is linearly unstable. For values of $k_{x}>32.3 \mathrm{~N} / \mathrm{mm}$ an unstable branch of periodic solutions bifurcates from the HP describing a subcritical Hopf-bifurcation as shown in Fig. 6c. This branch turns via a Fold Point (FP) at around $k_{x}=33 \mathrm{~N} / \mathrm{mm}$ into a stable branch of solutions. This stable branch of solutions can be continued for decreasing values of $k_{x}$. For values of $k_{x}<7.6 \mathrm{~N} / \mathrm{mm}$ the second HP leads also to a subcritical Hopf-bifurcation as presented in Fig. 6a. However, in this parameter range the unstable branch rises strongly in terms of $x_{\text {amp }}$ for decreasing values of $k_{x}$. After the steep rise of the unstable solution a fold bifurcation takes place at around $k_{x}=7 \mathrm{~N} / \mathrm{mm}$ at a comparatively high $x_{a m p}$, leading to a stable periodic solution. The stable branch of solutions decreases in $x_{a m p}$ with rising $k_{x}$ nearly as fast as the unstable branch of solutions had been growing. For further increasing values of $k_{x}$ the stable branch of the bifurcation diagram develops moderate amplitudes $x_{a m p}$ and joins with the branch that originates at the second FP at around $k_{x}=33 \mathrm{~N} / \mathrm{mm}$.

The polynomial type joint with a progressive, or stiffening, character leads to subcritical Hopf-bifurcations on both ends of the bifurcation diagram. Subcritical Hopf-bifurcations followed by a fold bifurcation are already well-known for friction induced flutter and can be provoked by a number of different nonlinearities, as e.g. nonlinear material properties [8]. However, an additional effect not discussed until now can be observed. In the subcritical regime for low values of $k_{x}$ the amplitude $x_{\text {amp }}$ of the stable periodic solution becomes extremely large, whereas for the most part of the parameter space the amplitude remains moderate. Taking into consideration that this deflection quantity is 
a)

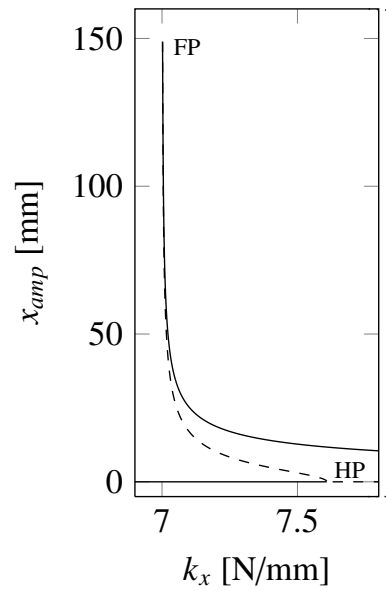

b)

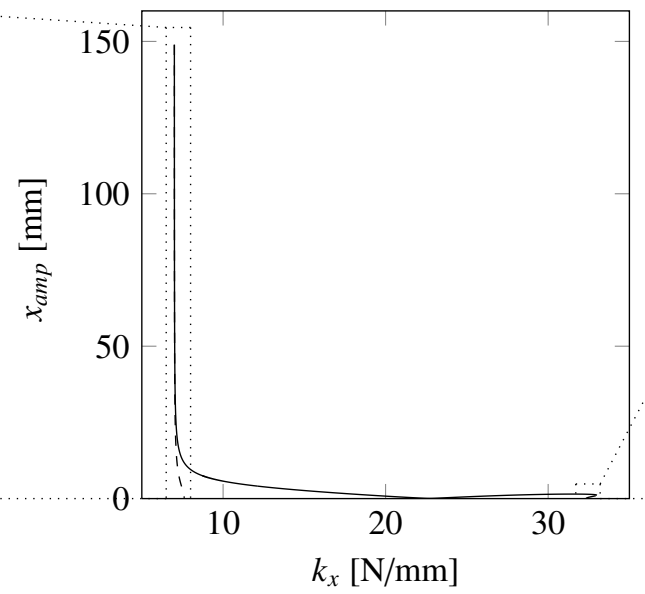

c)

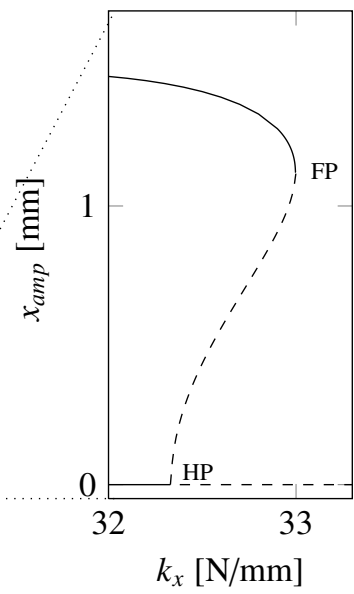

Figure 6: Bifurcation diagram with $k_{x}$ as bifurcation parameter. Stable equilibrium and periodic solutions are plotted as solid lines, unstable solutions as dashed lines. a) and c) show details of b) presenting the subcritical behaviour close to the HPs.

directly related to the amplitude of the limit cycle, it becomes clear that in this region the size of the eigenvalue's real parts from the stability calculation of the linearized system does not correlate with the limit cycle size at all. It seems that the nonlinear dynamics due to the joint properties dominate the amplitude development, and maximal limit cycle amplitudes can even appear in parameter ranges with a linearly stable equilibrium solution.

The reason for the effect of very large amplitudes for certain parameter combinations is directly related to the dynamics of the joint itself. Starting with small vibrational deflections, for which the linearized model should give reasonable results, the eigenvectors of the linearized system can be taken as a good starting point for the analysis of joint dynamics. A characteristic quantity capturing the dynamics of the joint in this linearized model system might be defined by the relative displacement in the joint. It can be characterized most easily as

$$
u_{E V}=\left\|-\left(\frac{\sqrt{2}}{2} \cdot x+\frac{\sqrt{2}}{2} \cdot y\right)+z\right\|,
$$

where the displacement components $x, y$ and $z$ can be taken from the unstable eigenmode under consideration. The quantity $u_{E V}$ is directly related to the strain and the strain energy of the joint. It will simply be referred to as joint strain in the following.

Fig. 7a combines the amplitude of the periodic solutions in $x$-direction and the joint strain $u_{E V}$ as defined in (5) for $k_{x}$ as the bifurcation parameter. The solutions show large amplitudes exactly for those parameters for which the joint is not strained according to the analysis of the linearized model. The finding seems intuitively convincing, since a certain level of joint strain is necessary in order to enable the joint nonlinearities to limit vibrational amplitudes. When the nonlinear joint is not strained due to the overall system dynamics, the joint is deactivated and stops being the decisive design element to limit the vibration amplitudes.

Following this observation, the results also mean that the parameter ranges for which large amplitudes appear can already be predicted from a simple analysis of the linearized system's vibration modes, since they seem to determine if a joint becomes an active element which restrains vibration amplitudes strongly, or not. In that case an additional post processing step in the analysis of the linearized model could be used to differentiate regions in the parameter space that lead to rather small vibration amplitudes from regions leading to large ones.

Of course, such a predictive approach will only be successful if the system under investigation is only weakly nonlinear in the sense that the nonlinear deflection shapes for finite amplitude vibration still resemble the linear modes. Indeed, also for the small present model cases where the nonlinear deflection shape deviates from the linear one do exist. However, even though the prediction based on the linear joint strain might then fail, the joint strain, now based 
a)

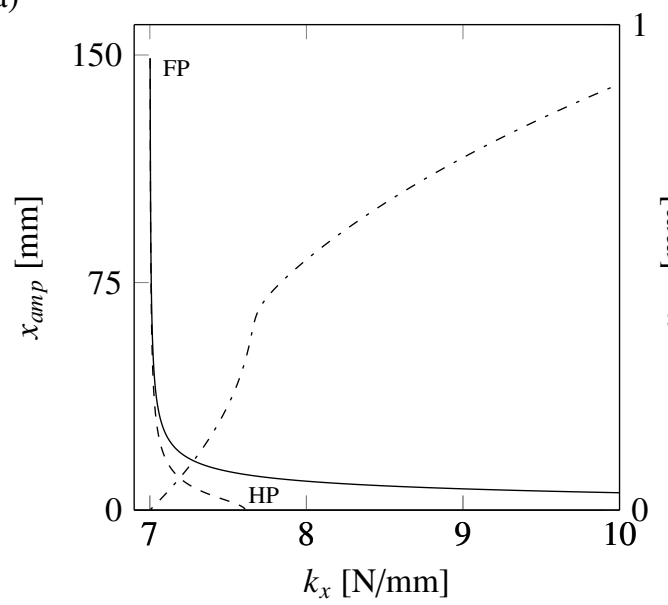

b)

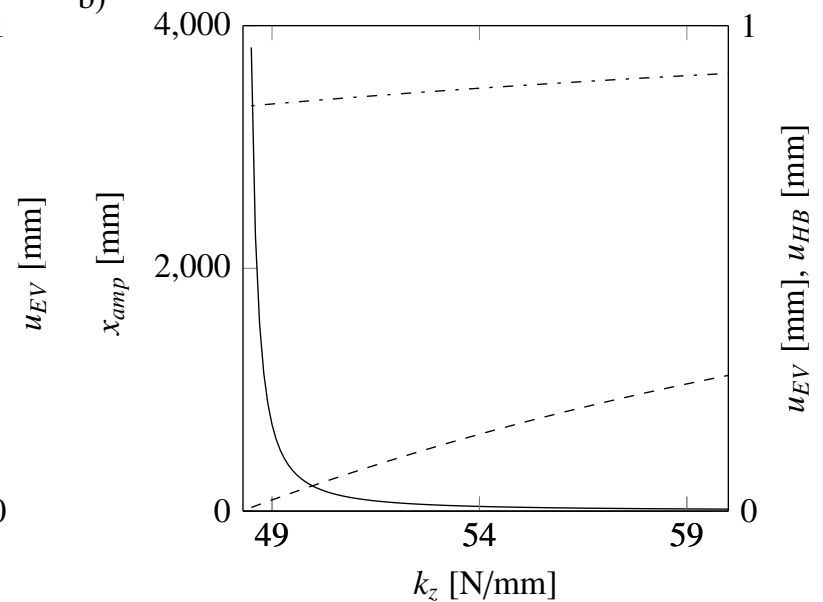

Figure 7: Bifurcation diagram with $k_{x}$ in a) and $k_{z}$ in b) as parameters. Stable periodic solutions are plotted as solid lines, unstable solutions as dashed lines. Additionally, the evolution of the normalized joint deflections $u_{E V}$ (dash-dot line) and $u_{H B}$ (dashed line) are given.

on the deflection of finite amplitude oscillation, still plays the pivotal role. To show this, a nonlinear joint strain $u_{H B}$ can be introduced as an extension of $u_{E V}$ into the nonlinear regime

$$
u_{H B}=\left\|-\left(\frac{\sqrt{2}}{2} \cdot \tilde{x}+\frac{\sqrt{2}}{2} \cdot \tilde{y}\right)+\tilde{z}\right\|,
$$

where $\tilde{x}, \tilde{y}$ and $\tilde{z}$ are based on the limit cycle deflections of the corresponding degrees of freedom obtained from HB. Hence, $u_{H B}$ represents the joint strain for the nonlinear deflection shape. This measure can differ significantly from the linear one $u_{E V}$, if the assumption of a system being weakly nonlinear is invalid. In this sense, the measure $u_{H B}$ is able to capture the joint's activity for a wider set of nonlinear systems than $u_{E V}$. Its determination, however, generates a larger numerical effort. Thus, the simpler measure for joint activity $u_{E V}$ based on the linear modes is advantageous in terms of computational effort.

Fig. 7b shows a representative example, employing $k_{z}$ as introduced in Fig. 1a as a bifurcation parameter. It turns out that again the parameter ranges of minimum joint strain, although now based on the deflections from HB, yield the maximum vibration amplitudes. So it is again the joint strain that dominates the amplitude behaviour, although now a nonlinear approximation is necessary to predict parameter ranges with large limit cycle amplitudes.

\subsection{Limitations of linear stability: bifurcation from infinity and detached limit cycles}

Now the model presented in Fig. 1b including the elasto-slip joint is studied. The variation of linear stiffness $k_{x}$ generates a bifurcation diagram very similar to the one using the smooth joint from Fig. 6 , but it is lacking the amplitude maximisation. Hence, the presentation in this work directly starts out by varying the nonlinear parameter of the nonsmooth joint represented by the tangential joint stiffness $k_{t}$ and the threshold force $F_{R}$ in the joint as introduced in equation (2). Beginning with a variation of the tangential joint stiffness, a low value of $k_{t}$ represents a joint with a rather low tangential stiffness, corresponding to a sliping threshold at comparatively large displacements, whereas a high value of $k_{t}$ can be interpreted as a stiff joint with a slipping threshold already at small displacements. With $k_{t} \rightarrow \infty$ the elasto-slip joint approaches the classical Coulomb-friction element leading to a differential inclusion of Fillipov type $[43,19]$. The results of this study are presented in Fig. 8.

The linearized model shows two HPs at around $k_{t}=4 \mathrm{~N} / \mathrm{mm}$ and $k_{t}=32 \mathrm{~N} / \mathrm{mm}$. Between these points again the system's equilibrium solution is unstable, i.e. the linearized system has a positive real part for one eigenvalue. For values $k_{t}<4 \mathrm{~N} / \mathrm{mm}$ and $k_{t}>32 \mathrm{~N} / \mathrm{mm}$ the system's equilibrium solution is stable. At $k_{t}=4 \mathrm{~N} / \mathrm{mm}$ a branch of unstable periodic solutions arises from the HP representing a subcritical Hopf-bifurcation as already present in section 3.1. Via a 
a)

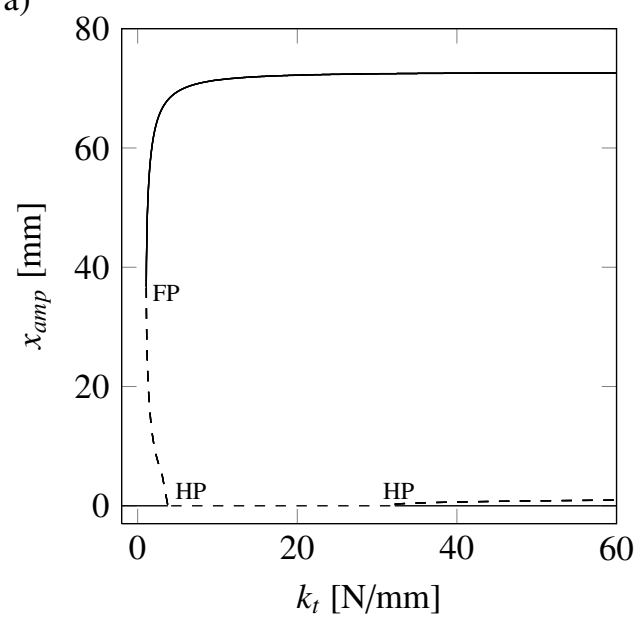

b)

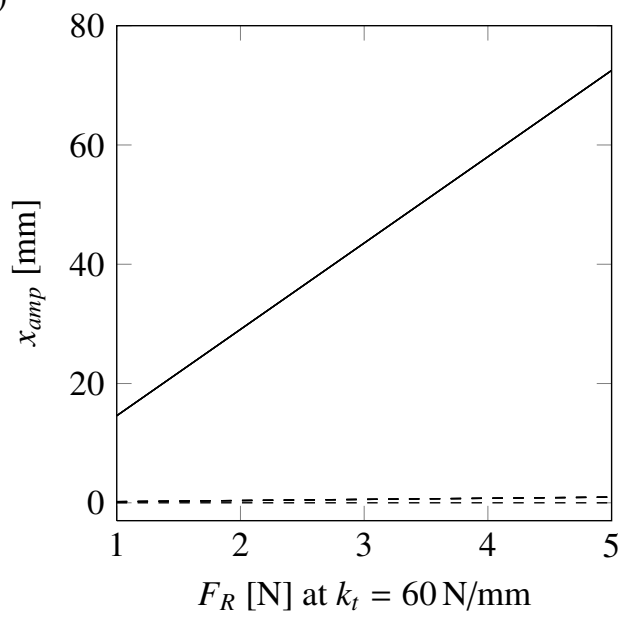

Figure 8: Bifurcation diagrams with $k_{t}$ in a) and $F_{R}$ in b) as bifurcation parameters. Stable equilibrium and periodic solutions are plotted as solid lines, unstable solutions as dashed lines.

FP at around $k_{t}=1 \mathrm{~N} / \mathrm{mm}$ this unstable branch turns into a stable one which after an initial growth is nearly unchanged in amplitude $x_{a m p}$ for larger $k_{t}$. At $k_{t}=32 \mathrm{~N} / \mathrm{mm}$ a similar scenario can be observed: an unstable periodic solution emanates from the HP. In contrast to the previous case where the subcritical Hopf-bifurcation is followed by a fold bifurcation, after an initial growth this branch of unstable periodic solutions remains at comparatively low levels of $x_{a m p}$ and evolves parallel to the branch of stable periodic solutions for $k_{t} \rightarrow \infty$.

Discussing the limit of $k_{t} \rightarrow \infty$, i.e. the pure Coulomb friction joint, it seems that besides the equilibrium solution, there exists an unstable periodic solution with small amplitudes, and a stable periodic solution with large amplitudes. In fact, a similar behaviour has recently been observed for friction induced vibrations including a dry friction element in $[19,20]$. The present study thus complements this finding and confirms it also for the context of friction induced flutter. Just for completeness one might note that the overall bifurcation structure could also be termed a global bifurcation from infinity (see e.g. $[44,45,46]$ ), since the branches of the periodic solution may be thought to originate at infinite values of $k_{t}$.

To further investigate the dependence of the results on the second elasto-slip joint parameter $F_{R}$, Fig. $8 \mathrm{~b}$ gives the amplitude $x_{a m p}$ of the solutions (at an arbitrary, but fixed value of $k_{t}=60 \mathrm{~N} / \mathrm{mm}$ ) vs. the threshold force $F_{R}$, which controls the onset of slipping in the joint. Obviously, the amplitudes of the periodic solutions depend linearly on $F_{R}$, which again confirms the finding of the periodic solutions bifurcating from infinity.

However, these results also raise the question, if some other parameter changes might transform the global bifurcation from infinity into a standard fold bifurcation linking the two branches of periodic solutions. It seems that this cannot directly be answered by a variation of $k_{x}$ or the elasto-slip joint parameters. Therefore, the level of damping is varied. Fig. 9 shows that increased damping, $d_{x}$ and $d_{y}$, can indeed destroy the bifurcation from infinity. Fig. $9 \mathrm{a}$ shows where the FPs and the HPs can be found when damping varies. For vanishing damping there is a bifurcation from infinity. For increasing values of $d_{x}$ and $d_{y}$ first a FP appears, and then approaches the HP (at $k_{t}=32 \mathrm{~N} / \mathrm{mm}$ in Fig. 8a). This indicates that the global bifurcation from infinity originates from the FP of a subcritical instability being moved out to infinity when damping decreases. At about $d_{x}=d_{y}=0.6 \mathrm{Ns} / \mathrm{mm}$ the FP vanishes as it collides with the $\mathrm{HP}$, which corresponds to the subcritical bifurcation turning over into a supercritical one. Fig. $9 \mathrm{~b}$ further clarifies this by showing that the FP approaches the HP in both dimensions $k_{t}$ and $x_{a m p}$. Thus, the global bifurcation from infinity turns via a subcritical bifurcation into a supercritical one for increased damping.

To summarise this part of the study, compared to super- and subcritical Hopf-bifurcations for the joint with nonlinear stiffness characteristics in section 3.1, an additional effect makes its appearance with the elasto-slip joint including dry friction: bifurcation from infinity. As a result an infinitely large area in the parameter space may exist where the equilibrium solution is linearly stable but stable limit cycle oscillations may co-exist. 
a)

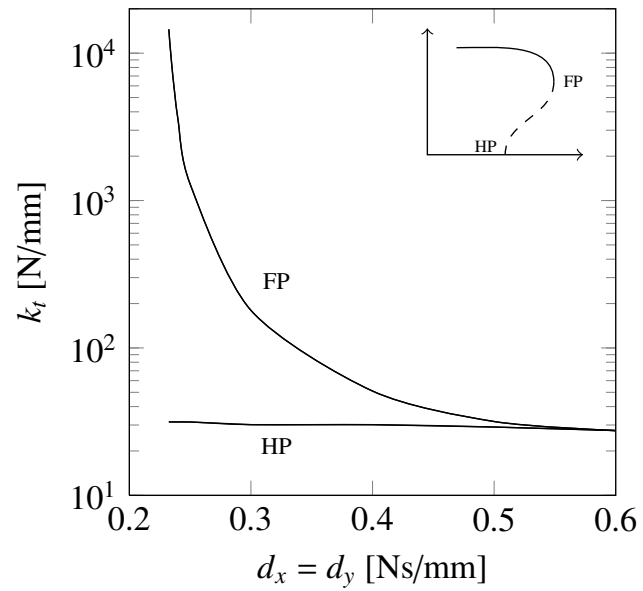

b)

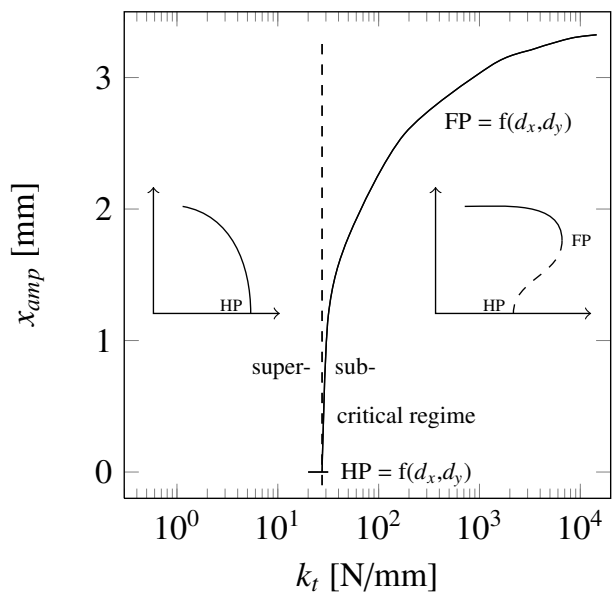

Figure 9: Evolution of FP and HP for growing values of $d_{x}$ and $d_{y}$ in terms of $k_{t}$ in a) as well as evolution of the FP in terms of vibration amplitude $x_{a m p}$ and bifurcation parameter $k_{t}$ as a function of $d_{x}$ and $d_{y}$ in b). Small schematic diagrams underline subcritical and supercritical bifurcation regimes.

But there is another remarkable effect: branches of isolated limit cycles, not bifurcating from the steady sliding state, sometimes also called isola, may appear. They can e.g. be generated by varying the linear stiffness $k_{x}$. For this purpose Fig. 10 shows a bifurcation diagram for the vibration amplitude $x_{a m p}, y_{a m p}$ and $z_{a m p}$ with $k_{x}$ as the bifurcation parameter at fixed value of $k_{t}=60 \mathrm{~N} / \mathrm{mm}$, situated clearly in the subcritical regime of Fig. 8a.

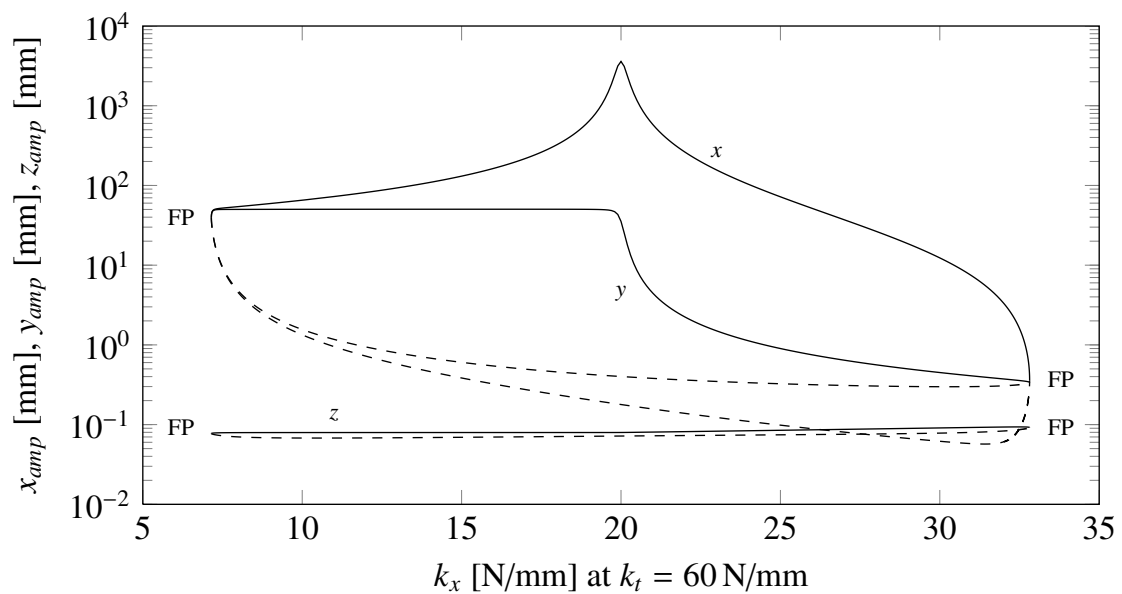

Figure 10: Bifurcation diagram with $k_{x}$ as bifurcation parameter. Stable branches are plotted as solid lines, unstable branches as dashed lines.

With this set of parameters the linearized model shows no unstable equilibrium solutions in the explored parameter space at all. However, detached periodic solutions, i.e. branches which are not connected to the equilibrium solution via HPs, coexist with stable equilibrium solutions. For values of $k_{x}<7 \mathrm{~N} / \mathrm{mm}$ the only existing solution is the stable equilibrium. For values from $k_{x}=7 \mathrm{~N} / \mathrm{mm}$ to $k_{x}=33 \mathrm{~N} / \mathrm{mm}$ three solutions to the problem can be found: beside the stable equilibrium solution there exist a stable and an unstable periodic solution. The branches of periodic solutions are connected via two FPs at $k_{x}=7 \mathrm{~N} / \mathrm{mm}$ and $k_{x}=33 \mathrm{~N} / \mathrm{mm}$. Between the two FPs the stable solutions show larger amplitudes $x_{a m p}, y_{a m p}$ and $z_{a m p}$ while the unstable solutions show smaller amplitudes. For the symmetric case of 
$k_{x}=k_{y}=20 \mathrm{~N} / \mathrm{mm}$ a resonance phenomenon in $x$-direction can be observed. For values of $k_{x}>33 \mathrm{~N} / \mathrm{mm}$, again, only the stable equilibrium solution exists.

Hence, for the analyzed minimal model including an elasto-slip joint detached periodic solutions, isola, may exist. These solutions are detached in the sense that their branches are not connected with the equilibrium solution through a bifurcation. Thus, a system with a steady sliding state that is linearly stable in the whole parameter range of interest might still be driven into limit cycle oscillation through finite disturbances.

The results for the elasto-slip joint show that the stability and bifurcation behaviour is strongly affected by the joint dynamics with its Coulomb characteristic. Linear stability analysis of the system may turn out to be of less significance or, in the worst case, even of no significance. The non-existence of linear stability borders for the linearized system when detached periodic solutions exist is the worst case scenario. In addition, hysteresis effects are likely to be expected when parameter variations take place, jump phenomena might be expected in parameter ranges of bi-stability. Due to the finite size of basins of attraction for solutions also background noise or other excitations might play a prominent role in predicting squeal occurrence.

\section{Summary, Conclusions and Outlook}

This work explores the influence of joints on stability and bifurcation behaviour of a system subject to friction induced flutter. It turns out that the dynamic state of the joints involved substantially decides about the size of the resulting limit cycle amplitudes. Very large amplitudes may especially arise for parameters where the joints are not active, either due to them being not activated already in the context of small amplitudes, i.e. the linearized system perspective, or due to being deactivated in the finite amplitude vibration state. It is also shown that subcritical Hopf-bifurcations, bifurcations from infinity and detached limit cycles, i.e. isola, may arise when joints including dry friction or elasto-slip characteristics are involved.

The results clearly indicate the possibly severe limitations of linear stability analysis based on eigenvalue calculation of a linearized model whenever joints are present. The size of the eigenvalues' real parts of a stability analysis, does usually not correspond in any sense to the size of the limit cycle amplitudes, when joints play a major role for the system dynamics. Moreover, the borders of stability predicted by linear analysis can become nearly meaningless when the nonlinear system dynamics are dominated by joints. This fact is exemplified by the possibility of bifurcations from infinity coming with negligibly small basins of attraction of the linearly stable sliding state or, even more severe, by detached periodic solutions. Hence, the application of linear stability analysis in the field of brake squeal and the interpretation of results needs to be executed very carefully. The presented limits of the linearization approach need especially to be kept in mind when dealing with joints. Nevertheless, deflection shapes based on the linearized system may be used for a first prediction of parameter ranges in which the limit cycle amplitudes will be very large, when the underlying system is weakly nonlinear as it seems to be the case quite often regarding brake squeal (e.g. [47]). However, the predictions should better be verified by double checking either with an analogous consideration based on HB methods, or a full limit cycle calculation. In that sense, and altogether, this study has thus presented further evidence that nonlinear analysis techniques are inevitable in order to develop numerical simulation tools towards predictive capabilities in brake vibrations and noise.

Definitely further analysis will be necessary to apply and extend the present findings to the large-scale models in use today. First of all, it will be necessary to reliably identify those joints in the multi-component, large- and multi-scale systems that dominate the nonlinear system behaviour and the amplitude of limit cycles. Second, the dynamics of joints itself need to be described and modelled in an improved way. The approximation of limit cycles is obviously limited by the quality of the joint models. The described techniques will only be successful if the models of joint dynamics improve. Third, the results need to be extended to systems showing multi-instabilities, what will allow a discussion of basins of attraction for distinct stable periodic solutions, as well as on the relevance of quasi-periodic solutions and mixed modes. Fourth, the techniques for limit cycle approximation themselves need to improve in both efficiency and accuracy, before they will be used in the day-to-day business of industrial engineering. Only when the methods of nonlinear analysis for friction induced vibration have become much more usable, they will spread into industry. 


\section{Appendix A. System matrices}

$$
\begin{aligned}
& \mathbf{M}=\left[\begin{array}{ccc}
M & 0 & 0 \\
0 & M & 0 \\
0 & 0 & m
\end{array}\right], \\
& \mathbf{D}=\left[\begin{array}{ccc}
d_{x} & 0 & 0 \\
0 & d_{y} & 0 \\
0 & 0 & d_{z}
\end{array}\right], \\
& \mathbf{K}=\left[\begin{array}{ccc}
k_{x} & -\frac{1}{2} \cdot k_{y} \cdot \mu & 0 \\
-\frac{1}{2} \cdot k_{y} \cdot \mu & k_{y} & 0 \\
0 & 0 & k_{z}
\end{array}\right], \\
& \mathbf{N}=\left[\begin{array}{ccc}
0 & & \\
\frac{1}{2} \cdot k_{y} \cdot \mu & 0 & 0 \\
0 & 0 & 0
\end{array}\right] .
\end{aligned}
$$

\section{Appendix B. Set of parameters}

Table B.1: Set of parameters for simulation and parameter studies

\begin{tabular}{c|c|c|c|c|c|c|c|c} 
Parameter & $M$ & $k_{x}$ & $k_{y}$ & $d_{x}, d_{y}$ & $\mu$ & $F_{\text {stat }}$ & $F_{R}$ & $k_{t}$ \\
\hline \hline Value & 1 & 11 & 20 & 0.02 & 0.65 & 0 & 5 & 10 \\
\hline Unit & $\mathrm{kg}$ & $\mathrm{N} / \mathrm{mm}$ & $\mathrm{N} / \mathrm{mm}$ & $\mathrm{Ns} / \mathrm{mm}$ & - & $\mathrm{N}$ & $\mathrm{N}$ & $\mathrm{N} / \mathrm{mm}$ \\
\hline & \multicolumn{7}{|c}{} \\
\hline Parameter & $k_{n l}$ & $d_{l n}$ & $m$ & $k_{z}$ & $d_{z}$ & $k_{l n}$ & $\alpha$ & \\
\hline \hline Value & 5 & 0.02 & 1 & 100 & 0.02 & 10 & $\frac{\pi}{4}$ & \\
\hline Unit & $\mathrm{N} / \mathrm{mm}$ & $\mathrm{Ns} / \mathrm{mm}$ & $\mathrm{kg}$ & $\mathrm{N} / \mathrm{mm}$ & $\mathrm{Ns} / \mathrm{mm}$ & $\mathrm{N} / \mathrm{mm}$ & - &
\end{tabular}

\section{Appendix C. Limit cycle approximation approach}

The limit cycle approximation is established in the frequency domain using a Harmonic Balance Method (HBM) with a finite number of harmonics. Dealing with self-excited vibrations in the present case the methodology has to be adapted to the fact that the frequency of the periodic solution in unknown. The employed approach is explained in the next section.

\section{Harmonic Balance Approach}

Starting from the equations of motion presented in section 2 ,

$$
\mathbf{M} \cdot \ddot{\mathbf{x}}(t)+(\mathbf{D}+\mathbf{G}) \cdot \dot{\mathbf{x}}(t)+(\mathbf{K}+\mathbf{N}) \cdot \mathbf{x}(t)+\mathbf{f}_{\mathrm{nl}}(\mathbf{x}, \dot{\mathbf{x}}, t)=\mathbf{f}_{\mathrm{ext}}
$$


a complex Fourier ansatz to approximate the solution $\mathbf{x}(t)$ with a finite number $m_{h}$ of harmonics which has the form

$$
\mathbf{x}(t) \approx \mathfrak{R}\left(\sum_{m=0}^{m_{h}}\left(\hat{\mathbf{x}}_{m} e^{i m \omega t}\right)\right)
$$

is used to describe the motion of the system. Here, the amplitudes $\hat{\mathbf{x}}_{\mathbf{m}}$ are complex valued. Accordingly, the nonlinear forces can be approximated by a Fourier ansatz containing the same number of harmonics,

$$
\mathbf{f}_{\mathrm{nl}}(\mathbf{x}, \dot{\mathbf{x}}, t) \approx \mathfrak{R}\left(\sum_{m=0}^{m_{h}}\left(\hat{\mathbf{f}}_{\mathrm{nl}, \mathrm{m}} e^{i m \omega t}\right)\right) .
$$

The determination of the complex Fourier coefficients $\hat{\mathbf{f}}_{\mathrm{n} l \mathrm{~m}}$ for a higher harmonic approach is done by the Alternating Frequency Time Domain method (AFT) [48]. The nonlinear forces are computed in the time domain using all considered harmonics for one period and the coefficients are computed numerically by evaluating the Fourier integrals. Inserting ansatz equation (C.2) into equation (C.1) the transition to the frequency domain can be accomplished and the system can be arranged in the form

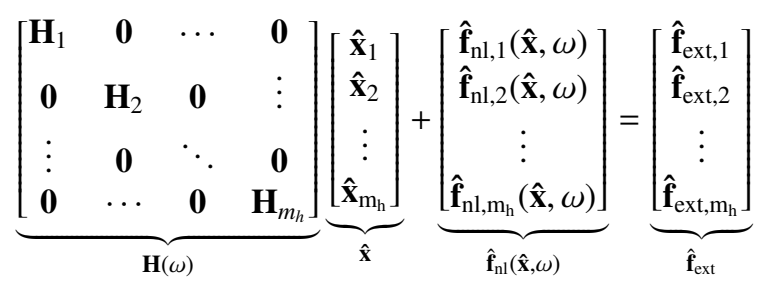

where the linear parts of the system are concentrated in the frequency dependent dynamic stiffness matrices

$$
\mathbf{H}_{m}(\omega)=(\mathbf{K}+\mathbf{N})+i m \omega(\mathbf{D}+\mathbf{G})-m \omega^{2} \mathbf{M} .
$$

Note, that in the application of the HBM for self-excited vibrations, there is no periodic excitation on the right hand side of the equation. The vector of the external forces $\mathbf{f}_{\mathrm{ext}}$ only contains the static force, which is applied to the system presented in section2. Since the nonlinear forces $\hat{\mathbf{f}}_{\mathrm{nl}}(\hat{\mathbf{x}}, \omega)$ depend on the solution $\hat{\mathbf{x}}$, the problem is rearranged in an implicit form

$$
f_{1}=\mathbf{H}(\omega) \hat{\mathbf{x}}+\hat{\mathbf{f}}_{\mathrm{nl}}(\hat{\mathbf{x}}, \omega)-\hat{\mathbf{f}}_{\text {ext }} \stackrel{!}{=} 0 .
$$

For self-excited vibrations, the frequency of the system is a priori unknown and determined by the system itself. This implies that the iterative problem, posed in equation (C.6), is under-determined and a numerical solution concludes with the trivial solution for $\omega=0$. For the additional unknown variable $\omega$ an additional equation need to be stated.

\section{Additional constraint equation}

The additional equation must contain the information of a periodic closed limit cycle trajectory to be able to reject the trivial solution in the iterative process. Following Coudeyras et al. [31], this can be achieved by arranging the system in a state space form

$$
\dot{\mathbf{Y}}=\mathbf{A Y}+f_{\mathrm{nl}}(\mathbf{Y})
$$

with the vector of the variables $\mathbf{Y}$ and a system matrix $\mathbf{A}$ containing the linear matrices,

$$
\mathbf{Y}=\left[\begin{array}{l}
\mathbf{x} \\
\dot{\mathbf{x}}
\end{array}\right], \mathbf{A}=\left[\begin{array}{cc}
\mathbf{0} & \mathbf{I} \\
-\mathbf{M}^{-\mathbf{1}}(\mathbf{K}+\mathbf{N}) & -\mathbf{M}^{-\mathbf{1}}(\mathbf{D}+\mathbf{G})
\end{array}\right] .
$$

The nonlinear forces are separated from the linear part and can be added in the form

$$
f_{\mathrm{nl}}=\left[\begin{array}{c}
\mathbf{0} \\
\mathbf{M}^{-1}\left(\mathbf{f}_{\mathrm{ext}}-\mathbf{f}_{\mathrm{nl}}(\mathbf{x}, \dot{\mathbf{x}}, t)\right)
\end{array}\right] .
$$


A Jacobian of the system, containing the linear system matrix $\mathbf{A}$ and the Jacobian of the nonlinear force vector $f_{\mathbf{n l}}$ can be established as

$$
\mathbf{J}=\mathbf{A}+\mathbf{J}_{\mathrm{nl}} \text {. }
$$

A direct calculation of the Jacobian of the nonlinear forces is not straightforward. Hence, its determination is done iteratively by minimizing the deviation $\Delta$ of the linear approximation of the nonlinear force for a periodic solution in the iterative process at once. This can be written as

$$
\Delta=f_{\mathrm{nl}}\left(\mathbf{Y}_{\mathrm{s}}(t)\right)-\mathbf{J}_{\mathrm{nl}} \mathbf{Y}_{\mathrm{s}}(t) \stackrel{!}{=} 0,
$$

where $\mathbf{Y}_{\mathrm{s}}(t)=\mathbf{Y}_{\mathrm{s}}(t+T)$ denotes a periodic solution, when the system exhibits limit cycles with the period $T$, depending on the angular frequency $\omega$ which is to be determined in the iterative process. The derivative of the nonlinear force vector $f_{\mathbf{n l}}$ to receive the Jacobian of the nonlinear forces $\mathbf{J}_{\mathrm{nl}}$ can be done numerically by a finite-difference method. With the Jacobian matrix of the nonlinear forces $\mathbf{J}_{\mathrm{nl}}$ the eigenvalues of the Jacobian $\mathbf{J}$ can be solved. Using the fact that the system performs limit cycles in the case that the real part of one eigenvalue is zero and all others are negative [31], the additional constraint equation can be formulated as

$$
f_{2}=\max (\mathfrak{R}(\operatorname{eig}(\mathbf{J}))) \stackrel{!}{=} 0,
$$

By minimizing functions $f_{1}$ and $f_{2}$ simultaneously towards zero in the iterative optimization process, the system is no longer under-determined. The additional constraint equation avoids that the trivial solution is found and the amplitudes of the considered harmonics can be computed. Since the frequency is a priori unknown special attention has to be paid when choosing the initial conditions to achieve convergence.

\section{Initial Conditions and Continuation}

As proposed by Sinou et al. [49], the periodic solution of a dynamic system can be approximated by

$$
\mathbf{Y}_{0}(t, p, \lambda)=p\left(\boldsymbol{\Phi} e^{\lambda t}+\widehat{\boldsymbol{\Phi}} e^{\widehat{\lambda} t}\right)
$$

where $\boldsymbol{\Phi}$ denotes the eigenvector of the unstable mode and $\lambda$ its corresponding eigenvalue. The complex conjugates are indicated by $\widehat{\boldsymbol{\Phi}}$ and $\widehat{\lambda} . p$ is a scaling factor. To generate efficient intial conditions $\mathbf{Y}_{0}$ is decomposed into Fourier coefficients. These coefficients and the imaginary part of the unstable eigenvalue are used as a set of initial conditions to determine the first point on the branch of periodic solutions. Starting at the HP or in its vicinity this approach leads to fast convergence.

However, isola, i.e. detached periodic solutions, in the linearly stable regime are also discussed in this contribution. Thus, an additional approach to determine initial conditions is necessary. In this case, decomposition of stable periodic solutions (obtained via time integration schemes for different initial conditions) into Fourier coefficients is used. The unknown limit cycle frequency $\omega$ is determined based on the period $T$.

Having found the solution for one parameter set, the bifurcation paramater is varied and the next iteration loop is started. The initial values for the subsequent step are estimated using natural continuation as depicted in Fig. C.11. 


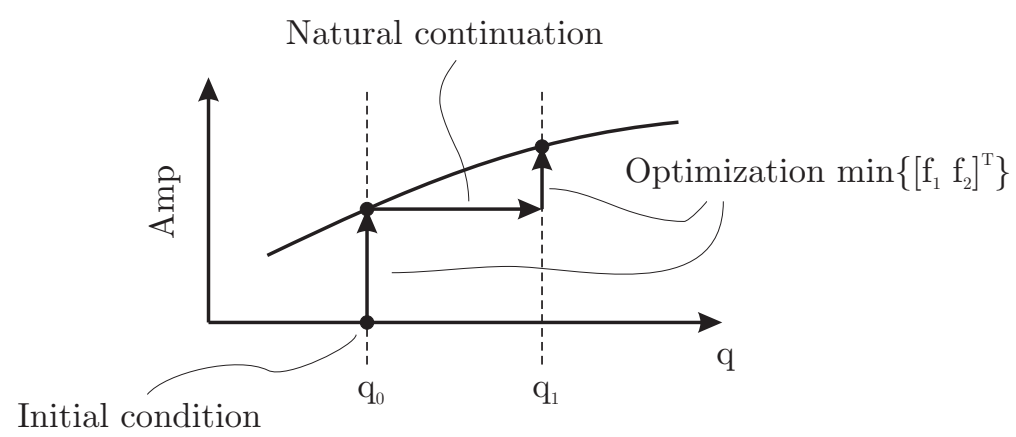

Figure C.11: Natural continuation scheme for a bifurcation parameter q

\section{References}

[1] N. North, Disc brake squeal, in: Proceedings of IMechE, no. 76 in C38, 1976, pp. 169-176.

[2] A. Papinniemi, J. Lai, J. Zhao, L. Loader, Brake squeal: a literature review, Applied Acoustics 63 (4) (2002) 391-400.

[3] N. M. Kinkaid, O. M. O'Reilly, P. Papadopoulos, Automotive disc brake squeal, Journal of Sound and Vibration 267 (2003) 105-166.

[4] G. Dihua, J. Dongying, A study on disc brake squeal using finite element methods, in: Proceedings of International SAE Congress and Exposition, 1998.

[5] S. Kung, K. Dunlap, R. Ballinger, Complex eigenvalue analysis for reducing low frequency brake squeal, Technical Report 2000-01-0444, SAE.

[6] H. Ouyang, W. Nack, Y. Yuan, F. Chen, Numerical analysis of automotive disc brake squeal: Numerical analysis of automotive disc brake squeal: a review, International Journal of Vehicle Noise and Vibration J. Vehicle Noise and Vibration 1 (3/4) (2005) 207-231

[7] A. AbuBakar, H. Ouyang, Complex eigenvalue analysis and dynamic transient analysis in predicting brake squeal, International Journal of Vehicle Noise and Vibration 2 (2006) 143-155.

[8] D. Hochlenert, Nonlinear stability of a disc brake model, Nonlinear Dynamics 58 (2009) $63-73$.

[9] D. Yuhas, M. Yuhas, Friction material elastic constant measurements, in: R. Quaglia, C. Tan, F. Chen (Eds.), Disc Brake Squeal- Mechanism, Analysis, Evaluation, and Reduction, SAE, 2005.

[10] M. Tiedemann, S. Kruse, N. Hoffmann, Dominant damping effects in friction brake noise vibration and harshness: the relevance of joints, Proceedings of the Institution of Mechanical Engineers, Part D: Journal of Automobile Engineering 0954407014536378, first published on June 9, 2014 as doi:10.1177/0954407014536378.

[11] M. Tiedemann, M. Stender, N. Hoffmann, Impact of joints on dynamic behaviour of brake systems, in: Proceedings of the Eurobrake 2014, 2014.

[12] K. Popp, Nichtlineare Schwingungen mechanischer Strukturen mit Kontakt- und Fügestellen, ZAMM - Zeitschrift für angewandte Mathematik und Mechanik 74 (3) (1994) $147-165$.

[13] L. Gaul, J. Lenz, Nonlinear dynamics of structures assembled by bolted joints, Acta Mechanica 125 (1997) 169-181.

[14] D. D. Quinn, D. Segalman, Using a series of iwan-type models for understanding joint dynamics, Journal of Applied Mechanics 72 (5) (2005) 666-673.

[15] D. J. Segalman, D. L. Gregory, M. J. Starr, B. R. Resor, M. D. Dew, J. P. Lauffer, N. M. Ames, Handbook of Dynamics of Jointed Structures, Sandia National Laboratories, 2009.

[16] L. Gaul, R. Nitzsche, The role of friction in mechanical joints, Applied Mechanics Review 54 (2) (2001) 93-106.

[17] S. Bograd, P. Reuss, A. Schmdit, L. Gaul, M. Mayer, Modeling the dynamics of mechanical joints, in: MSSP 25, Vol. 8, 2011, pp. $2801-2826$.

[18] C. Schwingshackl, E. Petrov, D. Ewins, Measured and estimated friction interface parameters in a nonlinear dynamic analysis, Mechanical Systems and Signal Processing.

[19] H. Hetzler, On the effect of nonsmooth coulomb friction on hopf bifurcations in a 1-dof oscillator with self-excitation due to negative damping, Nonlinear Dynamics 69 (1-2) (2012) 601-614.

[20] H. Hetzler, On the effect of non-smooth coulomb damping on flutter-type self-excitation in a non-gyroscopic circulatory 2-dof-system, Nonlinear Dynamics.

[21] H. Hetzler, Bifurcations in autonomous systems under the influence of joint damping, Journal of Sound and Vibration 333 (2014) 5933-5969.

[22] K. Popp, M. Rudolph, Vibration control to avoid stick-slip motion, Journal of Vibration and Control 10 (2004) 1585-1600.

[23] F. Massi, Y. Berthier, L. Baillet, Contact surface topography and system dynamics of brake squeal, Wear 265 (11) (2008) $1784-1792$.

[24] M. Eriksson, F. Bergman, S. Jacobson, Surface characteristic of brake pads after running under silent and squealing conditions, Wear 232 (1999) 621-628.

[25] F. Massi, L. Baillet, O. Giannini, A. Sestieri, Brake squeal: Linear and nonlinear numerical approaches, Mechanical Systems and Signal Processing 21 (6) (2007) $2374-2393$.

[26] J. Behrendt, C. Weiss, N. Hoffmann, A numerical study on stick-slip motion of a brake pad in steady sliding, Journal of Sound and Vibration 330 (4) (2011) 636-651.

[27] D. Tonazzi, F. Massi, L. Baillet, Y. Berthier, Instability scenarios between elastic media under frictional contact, Mechanical Systemes and Signal Processing 40 (2) (2013) $754-766$ 
[28] H. Hetzler, Zur Stabilität von Systemen bewegter Kontinua mit Reibkontakten am Beispiel des Bremsenquietschens, Ph.D. thesis, Technische Universität Karlsruhe, Karlsruhe (2008)

[29] G. V. des Roches, Frequency and time simulation of squeal instabilities, Ph.D. thesis, Ecole Centrale des arts et manufactures - Paris (2011).

[30] A. H. Nayfeh, D. T. Mook, Nonlinear Oscillations, Wiley Interscience, New York, 1979.

[31] N. Coudeyras, J. J. Sinou, S. Nacivet, A new treatment for predicting the self-excited vibrations of nonlinear systems with frictional interfaces: The constrained harmonic balance method, with application to disc brake squeal, Journal of Sound and Vibration (2009) 1175-1199.

[32] S.-W. Kung, V. C. Saligrama, M. A. Riehle, Modal participation analysis for identifying brake squeal mechanism, in: 18th Annual Brake Colloquium And Engineering Display, October 2000, San Diego, CA, USA, Session: NVH, 2000.

[33] N. Hoffmann, M. Fischer, A. R., L. Gaul, A minimal model for studying properties of the mode coupling type instability in friction induced oscillations, Mechanics Research Communications 29 (2002) 197-205.

[34] F. Massi, O. Giannini, L. Baillet, Brake squeal as dynamic instability: An experimental investigation, Journal of Acoustic Society America 120 (3) (2006) 1388-1398.

[35] N. Ghazaly, S. Mohammed, A. Abd-El-Tawwab, Understanding mode-coupling mechanism of brake squeal using finite element analysis, International Journal of Engineering Research and Applications Vol. 2 (1) (2012) 241-250.

[36] N. Hoffmann, L. Gaul, Effects of damping on mode-coupling instability in friction induced oscillation, ZAMM 8 (2003) 524-534.

[37] R. R. Craig, A review of time-domain and frequency-domain component mode synthesis method, in: Combined experimental/analytical modeling of dynamic structural systems; Proceedings of the Joint Mechnics Conference, American Society of Mechanical Engineers, 1985, pp. 11-39.

[38] E. Petrov, D. Ewins, Method for analysis of nonlinear multiharmonic vibrations of mistuned bladed discs with scatter of contact interface characteristics, in: ASME Turbo Expo 2004: Power for Land, Sea, and Air, American Society of Mechanical Engineers, 2004 , pp. $385-395$.

[39] E. Petrov, D. Ewins, Effects of damping and varying contact area at blade-disk joints in forced response analysis of bladed disk assemblies, Journal of Turbomachinery 128 (2) (2006) 403-410.

[40] E. Petrov, A high-accuracy model reduction for analysis of nonlinear vibrations in structures with contact interfaces, Journal of Engineering for Gas Turbines and Power 133 (10) (2011) 102503.

[41] N. Coudeyras, S. Nacivet, J. Sinou, Periodic and quasi-periodic solutions for multi-instabilities involved in brake squeal, Journal of Sound and Vibration 328 (2009) 520-540.

[42] A. Dhooge, W. Govaerts, Y. Kuznetsov, Matcont: A matlab package for numerical bifurcation analysis of odes, ACM Transactions on Mathematical Software 29(2) (2003) 141-164.

[43] R. Leine, H. Nijmeier, Dynamics and Bifurcations of Non-Smooth Mechanical Systems, Vol. 18 of Lecture Notes in Applied and Computational Mechanics, Springer Verlag, 2004.

[44] S. J. Cowley, F. Smith, On the stability of poiseuille-couette flow: a bifurcation from infinity, Journal of Fluid Mechanics 156 (83-100).

[45] M. Nagata, Three-dimensional finite-amplitude solutions in plane couette flow : bifurcation from infinity, Journal of Fluid Mechanics 217 (1990) 519-527 519-527 519-527 519-527.

[46] R. Seydel, Practical Bifurcation and Stability Analysis, Vol. 5 of Interdisciplinary Applied Mathematics, Springer Verlag, 2010.

[47] H. Marschner, D. Reckwerth, H. Steger, Innovative Schwingungsmesstechnik in der Bremsenentwicklung, Automobiltechnische Zeitschrift (ATZ) $110(2)$.

[48] T. Cameron, J. Griffin, An alternating frequency/time domain method for calculating the steady-state response of non-linear dynamic systems, Journal of Applied Mechanics.

[49] J.-J. Sinou, F. Thouverez, L. Jezequel, Stability analysis and non-linear behaviour of structural systems using the complex non-linear modal stability analysis and non-linear behaviour of structural systems using the complex non-linear modal analysis (cnlma), Computers and Structures 84 (29-30) (2006) 1891-1905. 